\title{
Mobile Agents: A Distributed Voice-Commanded Sensory and Robotic System for Surface EVA Assistance
}

\author{
William J. Clancey ${ }^{1}$, Maarten Sierhuis ${ }^{2}$, Rick Alena ${ }^{2}$, Sekou Crawford ${ }^{3}$, John \\ Dowding $^{2}$, Jeff Graham ${ }^{4}$, Charis Kaskiris ${ }^{5}, \mathrm{Kim} \mathrm{S}$. Tyree ${ }^{4}$, and Ronnie van Hoof ${ }^{2}$
}

\begin{abstract}
A model-based, distributed architecture integrates diverse components in a system designed for lunar and planetary surface operations: spacesuit biosensors, cameras, GPS, and a robotic assistant. The system transmits data and assists communication between the extra-vehicular activity (EVA) astronauts, the crew in a local habitat, and a remote mission support team. Software processes ("agents"), implemented in a system called Brahms, run on multiple, mobile platforms, including the spacesuit backpacks, all-terrain vehicles, and robot. These "mobile agents" interpret and transform available data to help people and robotic systems coordinate their actions to make operations more safe and efficient. Different types of agents relate platforms to each other ("proxy agents"), devices to software ("comm agents"), and people to the system ("personal agents"). A state-of-the-art spoken dialogue interface enables people to communicate with their personal agents, supporting a speech-driven navigation and scheduling tool, field observation record, and rover command system.

An important aspect of the engineering methodology involves first simulating the entire hardware and software system in Brahms, and then configuring the agents into a runtime system. Design of mobile agent functionality has been based on ethnographic observation of scientists working in Mars analog settings in the High Canadian Arctic on Devon Island and the southeast Utah desert (Clancey 2002a). The Mobile Agents system is developed iteratively in the context of use, with people doing authentic work. This paper provides a brief introduction to the architecture and emphasizes the method of empirical requirements analysis, through which observation, modeling, design, and testing are integrated in simulated EVA operations.
\end{abstract}

\section{Project Background}

The Mobile Agents project anticipates exploration of Mars, in which a crew of six people live in a habitat for many months. One long-term objective is to automate the

\footnotetext{
${ }^{1}$ NASA/Ames Research Center, Computational Science Division, MS 269-3, Moffett Field, CA 94035; Also Institute for Human and Machine Cognition, UWF, Pensacola, FL; William.J.Clancey@nasa.gov.

${ }^{2}$ NASA/Ames Research Center (Sierhuis \& Dowding: RIACS; van Hoof: QSS)

${ }^{3}$ Stanford University, Department of Mechanical Engineering, Stanford, CA

${ }^{4}$ NASA/Johnson Space Center, Houston, TX (Graham: Titan, Inc.)

${ }^{5}$ UC Berkeley, Economics, CA
} 
role of CapCom in Apollo, in which a person on Earth (in Houston) monitored and managed the navigation, schedule, and data collection during lunar traverses (Clancey, in press b). Because of the communication time delay this function cannot be performed from Earth during Mars exploration, and other crew members will often be too busy with maintenance, scientific analysis, or reporting to attend to every second of a four to seven hour Extra-Vehicular Activity (EVA) (Clancey in press a).

We have previously described how the Brahms simulation system (Clancey et al. 1998; Sierhuis 2001) has been adapted to provide both a tool for specifying multiagent systems and an implementation architecture for runtime agent interactions on mobile platforms (Clancey, et al. 2003). We have described how empirical studies at analog work sites, combined with studies of Apollo lunar traverses, provide automation ideas and metrics for developing tools and protocols for Mars exploration (Clancey 2002a; in press b). Previous publications have outlined two preliminary field tests at Johnson Space Center and Meteor Crater in 2002. We have emphasized that building a practical system in a difficult terrain prioritizes issues of network robustness and diminishes, at least initially, theoretical questions about agent competitiveness and cooperation.

The design of the Mobile Agents system has several theoretical dimensions. The overall design hypothesis is that automation can make EVAs more efficient and safe, with less human supervision than on Apollo. Partly automation is required by diminished mission support in a Mars mission configuration; partly it is enabled by digital media and telemetry (e.g., photos don't need to be physically returned on film). The evolving system is based on and contributes to theories of collaboration and dialogue management, particularly during field science and mission operations (Clancey in press b). Brahms itself is based on a theory of activities, that is, normbased practices by which tasks are actually accomplished (Clancey et al. 1998, $2002 \mathrm{~b}$ ). Finally, part of our research is the formalization of an engineering methodology that deliberately integrates historical and baseline studies, analog (simulation) expeditions, modeling, and iterative prototyping in authentic work settings.

\section{Mobile Agents April 2003 Test Configuration}

Mobile Agents is a distributed, multiple-agent system (cf. Pynadath \& Tambe 2003; Sycara, et al. 2003) in which agents are software programs in the Brahms activitybased language (Clancey 2002b). The agents contain and are linked by models that make "intelligent" operation possible. The agents are "mobile" because they are on movable platforms (backpacks, ATVs, robots). The models in the Mobile Agent architecture include:

- Agents representing people in the simulation system (used for testing the design protocols in pre-field test workflow simulations)

- Models of devices (e.g., camera) used in simulations

- Dynamic location model, including each agent and object (in terms of "areas" such as a habitat, and then specified by the Lat/Long coordination system)

- Network connectivity model, distributed in design of Comm and Proxy agents (respectively, relating external devices and agents to a local platform) 
- EVA Activity Plan: Sequence of activities specifying start and stop coordinate locations, duration, and thresholds allowed.

- Language model (Table 1): Word models and mapping of phrases to commands (with agent, location, object parameters)

- Command semantics, distributed in agent interactions-a work flow for communicating, accessing, and storing data (e.g., photographs, biosensors)

- Alarms, representing data thresholds (e.g., expected length of an activity within an EVA) and actions (e.g., where to communicate this information).

- ERA plans, locations, and control procedures (e.g., take photograph of location)

In preparation for the April 2003 test, the project team developed Brahms systems to run respectively on laptops located: 1) on the EVA Robotic Assistant ("ERA"; Shillcutt et al. 2002), 2) on spacesuit backpacks ("AstroBrahms"), and 3) in the Mars Desert Research Station habitat (MDRS hab) near Hanksville, UT ("HabCom"). The ERA model controls the ERA through voice commands (Figure 1). The two AstroBrahms systems provide astronaut data and voice command links to the rest of the system. The HabCom model, like the person it assists, monitors the EVA activity and biosensors worn by the astronauts. Platforms are connected on a wireless network (MEX-Alena et al. 2004; KAoS-Bradshaw, et al. 1997). The six biosensors are wired to an iPaq PDA worn by the astronaut, then transmitted by bluetooth to a Minibook computer attached to the top of backpack. A GPS unit, camera, and headphone-microphone are all connected to the Minibook. The ERA Brahms controlled the ERA's camera through an API to the ERA's computer. Language was developed using the RIALIST dialog system for each function to support natural spoken phrasings by the astronauts during the EVA (Table 1).

Based on previous experience in the Arizona monsoon, we chose to work in MDRS to have a permanent field shelter for working in April's rainy, cold, windy conditions (also sometimes dry and hot with dust storms). Satellite services from NASA/Glenn and NREN (Ames) provided an internet protocol telephone (NetPhone) to allow communications between support personnel at an EVA site and HabCom in MDRS.

The astronaut-geologists were provided with scripts to indicate the sequence of activities, including locations, and requested or optional commands to test. The astronauts could skip or repeat activities by indicating what they were doing (subject to predefined location and timing constraints). This was most useful for the authentic exploration at Lith Canyon, where Astro2 improvised a voice annotation (Table 2; Key: Activity Duration, Duration threshold in minutes, Location threshold in meters.)

The communication between people, software, and devices is quite complex (Figure 1): Astronauts and HabCom communicate via radio and NetPhone relay; people speak to their Personal Agents (PAs) on local computers using a microphone and receive feedback on a headset. PAs are communicating locally with external systems via "communications agents," providing an Application Programming Interface (API) to read data and control devices (e.g., camera). PAs (all implemented in Brahms on different computers) communicate via a wireless network (with repeaters) using the KAoS "agent registration" system. 
Table 1: Functionality tested April 2003 with representative voice commands

\begin{tabular}{|c|c|}
\hline Mobile Agents Functionality & Example Voice Command \\
\hline $\begin{array}{l}\text { Location Tracking } \\
\text { - } \quad \text { GPS mounted on backpacks \& ATVs } \\
\text { - } \quad \text { Flexible logging interval } \\
\text { - } \quad \text { Sent to Remote Science Team (RST) } \\
\text { at variable intervals } \\
\text { - Naming (predetermined vocabulary) }\end{array}$ & $\begin{array}{l}\text { start tracking my location every }<\mathbf{N}> \\
\text { seconds } \\
\text { call this location }<\text { location name }> \\
\text { where is }[<\text { location name }>\mid \text { Boudreaux]? }\end{array}$ \\
\hline $\begin{array}{l}\text { Science Data Logging } \\
\text { - Sample bags associated with } \\
\text { locations } \\
\text { - Voice annotations associated with } \\
\text { - } \quad \text { Pample bags and locations } \\
\text { - Stored at hab \& transmitted to RST } \\
\end{array}$ & $\begin{array}{l}\text { create sample bag \# } \\
\text { associate }\{\text { this }\} \text { [voice note|sample bag } \\
\{\#\} \text { ] with <location name }> \\
\text { record/play }\{\text { a }\} \text { voice note }\{\text { associated } \\
\text { with }<\text { location name }>\text { sample bag \#\} } \\
\text { upload [all | one] image }\{s\}\end{array}$ \\
\hline $\begin{array}{l}\text { Biosensor Logging } \\
\text { - } \quad \text { Transmitted via iPaq to backpack } \\
\text { - } \quad \text { Logged at intervals \& interpreted } \\
\end{array}$ & $\begin{array}{l}\text { start tracking my biosensors every } \\
<\mathrm{N}>\text { seconds }\end{array}$ \\
\hline $\begin{array}{l}\text { Activity Tracking } \\
\text { - Indicate start from predetermined list } \\
\text { - } \quad \text { Model of location, duration, sequence } \\
\text { - } \quad \text { Alerting for exceeding thresholds }\end{array}$ & $\begin{array}{l}\text { start <activity name> activity } \\
\text { how much time is left \{until next } \\
\text { activity\}? }\end{array}$ \\
\hline $\begin{array}{l}\text { ERA Commanding } \\
\text { - Move, follow, take a picture }\end{array}$ & $\begin{array}{l}\text { Boudreaux take a picture of [track } \\
\text { astronaut } \mid<\text { location name }>\text { ] }\end{array}$ \\
\hline
\end{tabular}

Table 2: Lith Canyon Scenario script (first half) provided to Astronauts.

\begin{tabular}{|c|c|}
\hline ACTIVITY PLAN & GEOLOGISTS' SCRIPT \\
\hline $\begin{array}{c}\text { <EVA PREP> } \\
(-,-,-)\end{array}$ & $\begin{array}{l}\text { 1. Drive in EVEREST with backpacks, helmets, suits, } \\
\text { all equipment } \\
\text { 2. Start Minibooks \& GPS } \\
\text { 3. Don suit with boots, gaiters, radios \& headsets } \\
\text { 4. Put on backpack \& helmet \& connect cables }\end{array}$ \\
\hline $\begin{array}{c}\text { Checking equipment } \\
(20,5,20)\end{array}$ & $\begin{array}{l}\text { 1. "Start CHECKING EQUIPMENT activity" } \\
\text { 2. "Start tracking my location every } 60 \text { seconds" } \\
\text { 3. "Start tracking my biosensors every } 5 \text { minutes" } \\
\text { 4. "Start WALKING TO TOP OF CANYON activity" }\end{array}$ \\
\hline $\begin{array}{l}\text { Walking to top of canyon } \\
(10,0,10)\end{array}$ & \{Astronaut 2 improvised a voice note during the walk \\
\hline $\begin{array}{c}\text { Sample fossils } \\
(10,5,0)\end{array}$ & $\begin{array}{l}\text { 1. "Start SAMPLE FOSSILS activity" } \\
\text { 2. Sample bag, voice annotation, association, photo } \\
\text { 3. "Start WALK TO HEAD OF CANYON activity" }\end{array}$ \\
\hline $\begin{array}{l}\text { Walk to head of canyon } \\
(10,0,10)\end{array}$ & $\begin{array}{l}<\text { Walk carefully down the hill and proceed to the head of } \\
\text { the canyon to the south (your left) }>\end{array}$ \\
\hline
\end{tabular}




\begin{tabular}{|c|c|c|c|c|}
\hline PEOPLE & $\begin{array}{l}\text { Microphone } \\
\text { \& \& } \\
\text { Headphone }\end{array}$ & $\begin{array}{c}\text { PERSONAL } \\
\text { AGENTS }\end{array}$ & $\begin{array}{l}\text { Comms } \\
\text { Agents }\end{array}$ & $\begin{array}{c}\text { Ext. } \\
\text { Systems }\end{array}$ \\
\hline Astro1 & $\leftarrow$ voice $\rightarrow$ & Agent $_{\text {AstroI }}$ & $\leftarrow \mathrm{API} \rightarrow$ & $\begin{array}{c}\text { GPS, ERA } \\
\text { Biosensors } \\
\text { Camera } \\
\end{array}$ \\
\hline$\frac{1}{\text { radio }}$ & & $\begin{array}{c}\mid \\
\text { wireless } \\
\text { network } \\
\mid\end{array}$ & & \\
\hline HabCom & $\leftarrow$ voice $\rightarrow$ & Agent $_{\text {HabCom }}$ & $\leftarrow \mathrm{API} \rightarrow$ & $\begin{array}{c}\text { Email (to } \\
\text { RST) }\end{array}$ \\
\hline $\begin{array}{c}\text { radio } \\
\mid\end{array}$ & & $\begin{array}{c}1 \\
\text { wireless } \\
\text { network } \\
\mid\end{array}$ & & \\
\hline Astro2 & $\leftarrow$ voice $\rightarrow$ & Agent $_{\text {Astro2 }}$ & $\leftarrow \mathrm{API} \rightarrow$ & $\begin{array}{c}\text { GPS, ERA } \\
\text { Biosensors } \\
\text { Camera } \\
\end{array}$ \\
\hline
\end{tabular}

Figure 1. Simplified schematic of MA configuration April 2003

\section{Scenarios and Results}

Six test scenarios were designed, ranging from a simple walk around the hab to a fullday drive onto a plateau. Actual testing involved incremental, pre-planned stages:

1) Wired test in the lower deck of MDRS to confirm communications protocols and peripheral connections

2) Wireless test inside the hab (without full suits, emphasizing communications and biosensors)

3) Test standing on the front porch of MDRS (allows use of GPS for first time)

4) Full walk (over one hour) around MDRS, following a script to test basic functionality; all systems running except ERA.

5) "Pedestrian EVA" with ERA, walking from porch to a dry wash about 100 meters south, gathering samples, taking photos, commanding ERA to take a photo, and return

6) "Lith Canyon EVA" involved two repeaters, ATV providing gateway to a LAN in the canyon, and a hour or more of scripted sampling and photography.

After two days of setup at and around MDRS, the scenarios were accomplished very gradually. Nearly 5 of 11 workdays were devoted to model modifications and testing inside MDRS. Functionality errors were discovered from incomplete end-toend simulation (caused by inadequate resources and planning). Repeatedly, we discovered complex interactions, such that every scenario constituted a new test, making perfection unimportant at each stage. For example, the GPS unit wouldn't work inside, and originally every scenario was based on a GPS signal being received before the first voice command was given. Not unexpectedly, network multi-pathing in the hills around MDRS caused erratic connections and high-volume data forwarding and alarms bogged down the network. Some missing capabilities that 
might have seemed advanced were actually key for testing; for example, starting the agent system required every platform to be running, complicating incremental tests.

Figure 2 shows the topography configuration for Lith Canyon; it required nearly two days to deploy communication equipment in preparation for this EVA. The Lith Canyon site involves broken ledges and steep cliffs $5 \mathrm{~km}$ from MDRS. The topography caused several serious problems:

- A wireless "shadow" occurred at the head of the canyon (as expected), causing the computerized backpacks to drop out of the network linking the astronauts back to MDRS. (Communications were properly queued and handled when possible.)

- The astronauts were unwilling to pass over a sharp, meter-high drop off in the canyon, requiring them to change the plan and walk around.

- The ERA was unable to follow the astronauts into the canyon because of the terrain, and even along the ledge had to be directly teleoperated with a person standing nearby.

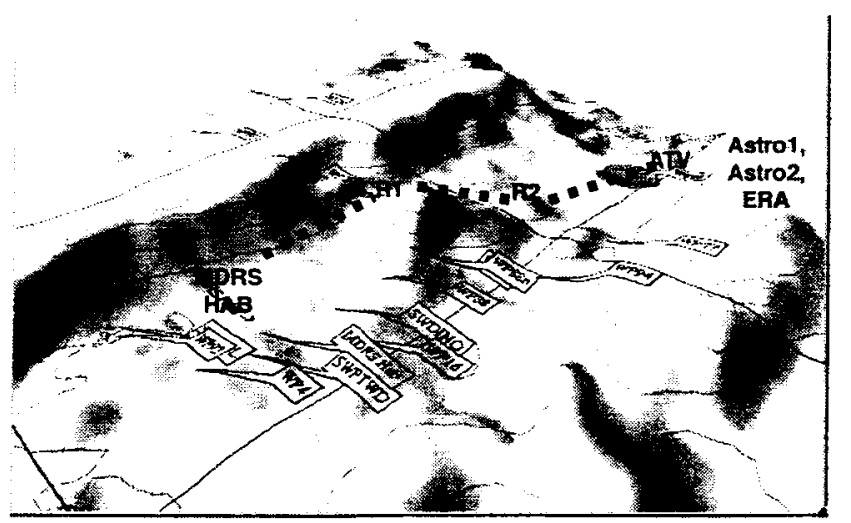

Figure 2. Topographic layout of MDRS (hab), Cisco repeaters, ATV LAN gateway, and astronauts with ERA in Lith Canyon (about $5 \mathrm{~km}$ from MDRS).

The Lith Canyon field test was a major accomplishment for the Mobile Agents project. The geologists' backpack computers running Brahms were wirelessly networked to another computer on an ATV $75 \mathrm{~m}$ away on a ledge across the canyon, and from there to a laptop running in MDRS more than $5 \mathrm{~km}$ away. The EVA lasted about 1.5 hours, as batteries allowed.

\section{Conclusions}

Field test results can be summarized according to lessons learned about the hardware, the agent architecture, and logistics of setting up the system and carrying out the scenarios:

- Hardware

- Technology required for field science is strongly topography driven

- A robot should be capable of working in terrain that geologists explore

- Need automated antenna and video tracking to monitor the astronauts

- Adapt ERA's differential GPS for astronauts

- Biosensors require more robust connectivity to backpack computers 
- Agent Architecture

- Copes well with loss of signal; but Astro PAs must take over some HabCom monitoring functions when nodes become isolated

- HabCom needs automated map to locate people, robot, ATVs and alarm log

- Need assertions to verify end-to-end functionality (e.g., RST received photo?)

- Voice feedback to positively confirm every command (e.g., "Do you want to start Checking Equipment activity?") and to indicate the status of processing was developed during the field modifications and proved highly beneficial

- Must integrate speech output, astronaut voice, and HabCom for recording

- Astronauts work in parallel, lack voice loop; they must coordinate to avoid

- Logistics work redundancy (e.g., need ability to record conversation as a "voice note")

- Need formal data network specification (GPS, computer, radio, biosensors)

- Need written specs/deliverables (not just design documents)

- Need field backups for all computers

Ideally, mobile agents should run for the duration of an entire mission-perhaps several years. However, agent interactions with the environment result in an everexpanding memory of beliefs. This can result in serious performance degradation of performance because of increased time in evaluating conditional actions. An interim solution, implemented during the field test, is to retract transitory communication beliefs (i.e., that a request has been received). A more systematic, built-in model of forgetting is required.

We learned a great deal about voice feedback, scenario design, network robustness, and system monitoring, exemplifying our approach of "empirical requirements analysis." One may design clever agent algorithms and architectures, but in practice simple services are needed that are never considered back in the home lab. In particular, we are frequently discovering new tools required to monitor and verify the system's operation during this developmental process. This phase can be expected to last many years, and involves viewing the agents as assisting in the research and ongoing redesign of the system.

As suggested by the previous tests, we conclude that a multiagent simulation with scenario-based formal specification accelerates cross-institution collaboration for integrating sensors, automation, procedures, and communications. We plan to return to MDRS in April 2004 to complete the scenarios with extended navigation and schedule monitoring, a medical agent to interpret bio-signs, augmented tools for the HabCom person to monitor the EVA, and a science database shared with the RST.

\section{Acknowledgments}

Funding is provided in part by the NASA/Ames Intelligent Systems Program, Human-Centered Computing area, managed by Mike Shafto. Besides the co-authors, our team includes a dozen other Brahms, ERA, and communications specialists. For related information (including our daily MDRS field reports), see http://bill.clancey.name \& http://www.agentisolutions.com. 


\section{References}

Alena, R., Ossenfort, J., Lee, C., Walker, E., Stone, T., in press. Design of Hybrid Mobile Communication Networks for Planetary Exploration. IEEE Aerospace Conference 2004.

Bradshaw, J. M., Dutfield, S., Benoit, P., and Woolley, J. D. 1997. KAoS: Toward an industrial-strength generic agent architecture. In J. M. Bradshaw (ed.), Software Agents, 375-418. Menlo Park, CA: AAAI Press.

Clancey, W. J., Sachs, P., Sierhuis, M., and van Hoof, R. 1998. Brahms: Simulating practice for work systems design. Int. J. Human-Computer Studies, 49: 831-865.

Clancey, W. J. 2002a. A Closed Mars Analog Simulation: the Approach of Crew 5 at the Mars Desert Research Station. Mars Society Annual Conference, Denver.

Clancey, W. J. 2002b. Simulating activities: Relating motives, deliberation, and attentive coordination. Cognitive Systems Research, 3(3) 471-499.

Clancey, W. J., Sierhuis, M., van Hoof, R., and Kaskiris, C. 2003. Advantages of Brahms for Specifying and Implementing a Multiagent Human-Robotic Exploration System. Proc. FLAIRS-2003, 7-11.

Clancey, W. J. in press a. Automating CapCom: Pragmatic Operations and Technology Research for Human Exploration of Mars. In C. Cockell (ed.), Martian Expedition Planning. AAS Publishers.

Clancey, W. J. in press b. Roles for Agent Assistants in Field Science: Understanding Personal Projects and Collaboration. IEEE Transactions on Systems, Man and Cybernetics. Issue on Human-Robot Interaction.

EVA Robotic Assistant. URL http://vesuvius.jsc.nasa.gov/er_er/html/era/era.html.

Pynadath, D. N., and Tambe, M. 2003. An Automated Teamwork Infrastructure for Heterogeneous Software Agents and Humans. Autonomous Agents and MultiAgent Systems, 7, 71-100.

Shillcutt, K., Burridge, R., Graham, J. 2002. Boudreaux the Robot (a.k.a. EVA Robotic Assistant). AAAI Fall Symp. on Human-Robot Interaction, Tech Rpt FS02-03, 92-96. Menlo Park, CA: AAAI Press.

Sierhuis, M. 2001. Modeling and simulating work practice. Ph.D. thesis, Social Science and Informatics (SWI), University of Amsterdam, The Netherlands, ISBN 90-6464-849-2.

Sierhuis, M. Clancey, W. J., and van Hoof, R. 2003. Brahms: A Multiagent Modeling Environment for Simulating Social Phenomena. First Conference of the European Social Simulation Association (SIMSOC VI), Gronigen, The Netherlands.

Sycara, K., Paolucci, M., Velsen, M. v., and Giampapa, J. 2003. The RETSINA MAS Infrastructure. Autonomous Agents and Multi-Agent Systems, 7, 29-48. 